\title{
3. Die Forschung
}

\subsection{Reiseberichte}

Seit der Kreuzfahrer-Zeit existiert eine Fülle von Reiseberichten über das Rauhe Kilikien. ${ }^{21}$ Erwähnung findet die Gegend bereits bei einigen mittelalterlichen Pilgern wie Wilbrand von Oldenburg, der 1211 die Gegend auf der Rückreise von Jerusalem bereiste und auch Seleucia an der Küste besuchte. ${ }^{22}$

Der britische Offizier William LEAKE bereiste Anfang des 19. Jahrhunderts als erster westlicher Besucher die isaurische Dekapolis. Die im Hochtaurus gelegene Polis Isaura wurde am 12. August 1837 von dem Kleriker William HAMILTON (1842) entdeckt. Charles TexIER (1834) bereiste die Gegend etwa zur gleichen Zeit; seine größte Entdeckung war die hethitische Haupstadt Hattusa. Zuvor hatte der gelehrte englische Fregattenkapitän Francis BEAUFORT für die Jahre 1811 bis 1812 von der britischen Admiralität den Auftrag zur Erforschung der kleinasiatischen Küsten erhalten und publizierte einen Atlas. Sein Prosawerk beschreibt die südliche Küstenlinie der Türkei; er gibt einen detaillierten Plan des Theaters von Side und beschreibt auch Seleucia (223 ff.) und Elaiussa Sebaste (249-253).

Der französische Orientalist Victor LANGLOIS bereiste entgegen der Angabe in seinem Titel Voyage dans la Cilicie et dans les Montagnes du Taurus im Jahr 1861 nicht das Hinterland im Taurus, sondern nur die Küstenlinie. Das Buch ist in ein 100seitiges Reisejournal und einen 300-seitigen wissenschaftlichen Teil gegliedert, worin sich die Kopien zahlreicher Inschriften und einige Zeichnungen von Ruinenstätten finden. Eine kurze Notiz zu Isaurien findet sich auf den Seiten $478 \mathrm{f}$.

Edwin J. Davis (1879) gibt einen Bericht aus der Sicht des interessierten Laien, zumal die im vorigen Jahrhundert geschilderten traditionellen Agrargebräuche seit der Antike unverändert waren. Der Amerikaner Sitlington STERRETT unternahm 1884 eine ausgedehnte Forschungsreise und sammelte zahlreiche Inschriften. J. Theodore BENT (1891) untersuchte die Ebene um Seleucia, Elaiussa-Sebaste, Korykos und Olba, und publizierte zahlreiche Inschriften. Karl Graf LANCKORONSKI (1890-1892) bereiste Lykien, Pisidien und Pamphylien mehrmals seit 1882 und gibt in der Einleitung zum ersten Band einen kleinen Reisebericht. In ihrem mit zahlreichen Photographien bestückten Reisebericht setzt Gertrude Lowthian BELL (1906-1907) den

21 Eine Liste von Reiseberichten gibt Mutafian (1988) 394-402; eine Bibliographie der Erkundungen bis zum Jahr 1903 SCHAFFER (1903) 98.

22 J. C. M. LAurent (ed.), Peregrinatores medii aevi quatuor, Leipzig 1864, I 26 p. 180. 
Schwerpunkt auf die Kirchenbauten. Wertvoll ist er heute noch durch die Abbildungen, die viele Bauten noch in einem besseren Zustand als dem heutigen zeigen.

\subsection{Epigraphische und archäologische Forschungen}

Das reichhaltige epigraphische Material wurde im Auftrag der Österreichischen Akademie der Wissenschaften gesichtet. ${ }^{23}$ Auch britische Forscher haben einige Expeditionen unternommen. ${ }^{24}$ Die geographische Erforschung Isauriens, die zu Beginn des 19. Jhs. mit den Reisen englischer Offiziere begonnen hatte, faßt erstmals 1890 William RAMSAY in seiner Historical Geography of Asia Minor zusammen. In seiner Social Basis of Roman Power in Asia Minor verzeichnet er Grabinschriften isaurischer Soldatenfamilien. In den 1960er Jahren haben George E. BEAN und Terence B. MITFORD das Rauhe Kilikien bereist und eine Vielzahl an Inschriften gesammelt. ${ }^{25}$ Kurt TOMASCHITZ von der Kleinasiatischen Kommission der Österreichischen Akademie der Wissenschaften übernahm seit 1989 das von Mitford hinterlassene Material für die Titulae Asiae Minoris ${ }^{26}$ und hat zusammen mit Stefan HAGEL 1998 ein vollständig indiziertes Sammelbuch aller westkilikischen Inschriften vorgelegt. Gertrud LAMINGER-PASCHER erstellte in den Jahren 1973 und 1974 einen Index Grammaticus zu den griechischen Inschriften Kilikiens und Isauriens. Die ältere Literatur bis 1970 über Kleinasien unter römischer Herrschaft faßte Thomas PEKÁRY 1980 zusammen.

Friedrich HILD und Hansgerd HeLlenKemPER veröffentlichten 1990 den Band „Kilikien und Isaurien“ in den Tabula Imperii Byzantini der Österreichischen Akademie der Wissenschaften, worin nach einer 50-seitigen Einleitung zu Geschichte und Geographie für jede isaurische Siedlung ein Lemma aufgeführt ist, das Geographie, Geschichte und Quellenlage umfaßt. Die jüngst erschienene Monographie von Stephen HILL über die isaurischen Kirchenbauten gründet sich auf die in zahlreichen Veröffentlichungen von Michael GoUGH in den Anatolian Studies geleisteten Vorarbeiten.

23 Adolf Wilhelm / Josef KeIL, Vorläufiger Bericht über eine Reise in Kilikien. Wien 1915; Rudolf Heberdey / Adolf Wilhelm, Reisen in Kilikien. 1896. (Österr. AdW, phil.-hist. Cl., 44, Nr. 6). Wien 1896; MAMA II; MAMA III.

24 BeLl (1906-1907); Gough (1954); WE.

25 BM I; II.

26 DOBESCH (1993) 27. 


\subsection{Großräumige Untersuchungen}

Die Isaurier finden bereits vielfältige Erwähnung in Edward GiBBoNs 1776-1788 erschienener History of the Decline and Fall of the Roman Empire, worin die von der Historia Augusta und Ammian geschilderten Vorkommnisse, die Regierung Zenos und der Krieg gegen die Isaurier unter Anastasius wiedergegeben sind. In nahezu allen gängigen Gesamtdarstellungen der Spätantike haben die Isaurier ihren Platz; auch die entsprechenden neuen Bände der Cambridge Ancient History widmen ihnen zahlreiche Einträge. $^{27}$

Das nach wie vor unübertroffene Standardwerk zur römischen Verwaltung in Kleinasien stammt von David MAGIE aus dem Jahr 1950; das jüngste Kompendium über Kleinasien in der hohen Kaiserzeit hat Maurice SARTRE 1995 verfaßt. Die Listen der römischen Statthalter in Kleinasien bis zu Diokletian hat kürzlich Bernard RÉMY publiziert. Ein die Gesamtgeschichte Kilikiens behandelndes Überblickswerk bietet Claude MUTAfIAN, eine Bibliographie über Kilikien bringt Marcello Spanu. ${ }^{28}$ Allgemein gehalten und sehr informativ, mit einer Fülle von neuer Literatur und zahlreichen Einzelaspekten, jedoch mit einigen problematischen, noch zu diskutierenden Thesen, sind die Beiträge von Stephen Mitchell.

\subsection{Einzeluntersuchungen}

Die Einzeluntersuchungen zu den Isauriern lassen sich in drei Themenbereiche gliedern: Romanisierung und Revolte während der gesamten Zeitspanne, die Erforschung der Aufstände im 3. und 4. Jahrhundert, und die Spätantike, vor allem das 5. Jahrhundert.

Für die erste Epoche sind die Arbeiten von Ronald SYME maßgeblich. Über die Kampagnen des P. Servilius Isauricus hat Paul T. KEYSER (1997) eine informative Arbeit veröffentlicht. Die am häufigsten zitierte Studie über Isaurien im 4. Jahrhundert ist jene von Jean RoUGÉ (1966), gefolgt von mehreren Artikeln von Ronald SYME. Rougés Anliegen war es vor allem, die Erzählungen der Historia Augusta zu Ereignissen in Isaurien zur Zeit der Kaiser Gallienus, Claudius Gothicus und Probus als Rückprojektionen aus dem 4. Jahrhundert zu erweisen. Gabriele MARASCO argumentiert ganz im Sinne RouGÉs, dagegen wenden sich nach den Forschungen von Mitchell zu Cremna Martin ZimmermanN und Hartwin BRANDT; sie verweisen auf inschriftlich bezeugte Gegenmaßnahmen von Polisbewohnern in Pamphylien und Pisidien, welche auf eine umfassende Tätigkeit von isaurischen Räubern bereits im 3. Jahrhundert hindeuten.

27 Seeck (1921); Stenn (1928) u. (1949); Demandt (1989); CAH XIII (1998), XIV (2000).

28 EQUiNi SCHNEIDER (1999) 405-439. 
Eine Reihe von kurzen Artikeln hat Keith HOPWOOD publiziert. Er betont darin die Balance zwischen Hirten und Seßhaften im Machtgefüge der Poleis. Inwieweit eine derartige Art von Beziehungsgeflecht allerdings noch für das 4. Jahrhundert gegolten hat, als die isaurischen Aufstände sich schon über das gesamte östliche Mittelmeer erstreckten, soll in dieser Arbeit diskutiert werden. Mit dem Banditenwesen im Allgemeinen und dem der Isaurier im Besonderen hat sich Brent D. SHAw in zwei langen Artikeln auseinandergesetzt. Er vertritt die These, daß das isaurische Bergland niemals hellenistischer, römischer oder byzantinischer Kontrolle unterlag. Auch Clifford E. MINOR bezeichnet die isaurischen Aufstände als „Proto-Nationalismus“. Dagegen wendet sich neuerdings Noel LENSKI in zwei wegweisenden Artikeln. Er widerlegt $u$.E. überzeugend SHAWs These von einem ewigen Widerstand der Isaurier und weist auf die schon früh und vor allem während der Hohen Kaiserzeit erfolgten hohen Romanisierungsgrad der Gegend hin. Erst mit der Reichskrise des 3. Jahrhunderts seien die Isaurier in den Widerstand zurückgefallen, was auch schon Ariel LEWIN in der Kilikien gewidmeten Nummer der Quaderni Storici in einem historischen Durchmarsch durch die Geschichte Isauriens herausgearbeitet hat.

Auch in dieser Arbeit soll gegen die verkürzende und ein gewisses Quantum an Sozialromantik enthaltende Sicht argumentiert werden, in Isaurien habe es sich um eine wie auch immer geartete ,nationale“ Bewegung gehandelt oder eine Alternative zum „Prinzip Rom“.

Die Machtverhältnisse und die Kämpfe Zenos mit den Goten auf dem Balkan, die vor allem bei Malchus geschildert sind, untersuchten Malcolm ERRINGTON und Peter HEATHER. Zuletzt schrieb Umberto ROBERTo (2000) über Candidus und das Aufstiegsbestreben der Isaurier; er hatte jedoch die Arbeiten von LENSKI noch nicht zur Kenntnis nehmen können. Brian CROKE (2001) untersuchte die Chronik des Marcellinus Comes und dessen Schilderung der isaurischen Machtkämpfe. Die Bedeutung der Isaurier als Volk versucht jüngst Hugh ELTON in mehreren Artikeln zu relativieren.

An Studien über den Kaiser Zeno (474-491) ist der noch immer zitable, grundlegende Aufsatz über Zeno und die Isaurier von Ernest W. BRooks aus dem Jahr 1893 zu nennen. Die 1894 erschienene Dissertation über Zeno von Wilhelm BARTH verarbeitet zwar alle damals bekannten Quellen (die zenofreundliche Vita des Säulenheiligen Daniel gehörte noch nicht dazu), jedoch zumeist deskriptiv. Da BARTH auch der Gewichtung folgt, welche die Quellen gesetzt haben, nehmen den größten Raum die romanhaften Schilderungen der Kämpfe gegen Zenos isaurischen Rivalen Illus ein. Der Autor versucht jedoch, die Politik seines Kaisers entgegen der hauptsächlich zenofeindlichen Überlieferung der Quellen in gutem Licht erscheinen zu lassen. Überhaupt zeigt das von Zeno vor allem in der älteren Forschung gezeichnete Bild, wie die sich widersprechenden Urteile aus den oftmals tendenziösen Quellen unkritisch übernommen werden. 
Die letzte umfangreiche Arbeit über Zeno ist Adolf LiPPOLDs Artikel in PaulyWissowas Realencyclopädie von 1972. Er bemerkte dort: „Die einst von BURY getroffene Feststellung, daß Zeno nie Favorit der Historiker gewesen ist, besitzt noch immer Gültigkeit". ${ }^{29}$ Dies ist zwar nur noch eingeschränkt richtig, doch 10 Jahre nach diesem Artikel wurde beispielsweise angemerkt, die interpretative Literatur zu Zenos Beziehungen zu den Balkangoten sei „nicht sehr umfangreich““. ${ }^{30}$ Auch die außenpolitischen Implikationen von Zenos religionspolitischer Einheitsformel, dem Henotikon, sind im Hinblick auf die mangelnde Akzeptanz der Isaurier in der römischen Welt von Interesse. Die Religionspolitik Zenos ist nach den umfangreichen Arbeiten von Eduard SCHWARTZ einer Dissertation von Benno SCHWARK aus dem Jahr 1950 behandelt; zuletzt mit noch zu diskutierenden Thesen im Jahr 1998 von Hanns Christof BRENNECKE.

Eine weitere in der Forschung diskutierte Frage ist, ob es die Isaurier als eigenes Volk überhaupt von Beginn an gegeben hat oder ob damit ein Völkergemisch bezeichnet wurde. ${ }^{31}$

\subsection{Erwähnung in Sammelwerken, Lexikonartikel}

Der Artikel von Wilhelm RugE in der Realencyclopädie über die Isaurier gilt als veraltet. $^{32}$ Im Neuen Pauly stammen die einschlägigen Artikel zu den Isauriern und deren Städten von Kurt TOMASCHITZ. ${ }^{33}$ Hervorragend, doch hauptsächlich unter theologischem Aspekt, ist jener über Zeno von Klaus-Peter TODT im BiographischBibliographischen Kirchenlexikon. ${ }^{34}$ Auch der erwähnte Artikel über Zeno von Adolf LIPPOLD aus dem Jahr 1972 ist nicht zu vergessen. Das Oxford Dictionary of Byzanti$u m$ gibt ebenfalls einen knappen Überblick; der Verfasser des Artikels geht jedoch zu weit, wenn er behauptet, die Isaurier seien im 5. Jahrhundert so weit integriert gewe-

29 LiPPOLd (1972) 213; BURY (1923) I 252; ähnlich STEIN (1949) 75 und JONEs (1964) 224. Bezuiglich der Einbindung von Zenos Zeit in einen größeren Rahmen bemerkte KAEGI (1968) VII in seiner Arbeit über Byzanz und den Niedergang Roms, the absence of a real discussion of Byzantine political and intellectual reactions to the decline, and disappearance of the Westem Roman Empire." Zum Verdikt der Quellen und der Forschung über den Charakter Zenos s.u. Kap. V.3.1.

30 ERrington (1983) 96, Anm. 28.

31 Holl (1908); RougÉ (1966) 282; Thompson (1946) 28; RAMSAy (1967) 231; Burgess (1990); s.u. II.5.

32 Walter Ruge, s.v. 'Isaura', $R E$ IX 2 (1916), $2055 f$.

33 Kurt Tomaschitz, s. v. 'Isauria, Isauroi' in: DNP 5 (1998), 1118.

34 Klaus-Peter TODT, s.v. 'Zenon' in: $B B K L$ XIV (1998) 418-427. 
sen, daß sie zu dieser Zeit den Kern der römischen Armee gebildet hättenn. ${ }^{35}$ Von geringem Informationsgehalt ist der entsprechende Eintrag in Late Antiquity. ${ }^{36}$

\subsection{Rezeption}

Die Lektüre von Jacob BURCKHARDTs Die Zeit Constantins des Großen veranlaßte im Jahr 1939 Gerhart HAUPTMANN zu dem Dramenfragment „Die Isaurier“ ${ }^{37}$ Es wurde erstmalig durch den Berliner Germanisten Peter SPRENGEL im Jahr 1999 publiziert $^{38}$ und interpretiert die bei BURCKHARDT aus Zosimus und der Historia Augusta geschilderten Ereignisse als den Rückfall eines vormals kultivierten Volkes in die Barbarei. Isaurien, so schon BURCKHARDT, „bildet in allen Geschichten der römischen Kaiserzeit einen stehenden Artikel.“ In Hauptmanns Exemplar des Buches ist der Satz „Isaurien ist hellenisiert gewesen, wenigstens oberflächlich, und hat sich später wieder allmählich barbarisiert" angestrichen, und die isaurische Stadt Germanicopolis ist mit der Randnotiz „sic“ gekennzeichnet. Dies kann als ein Versuch des ansonsten durch seine eher zustimmende Haltung gegenüber dem Nationalsozialismus schließlich in die Kritik geratenen naturalistischen Schriftstellers gedeutet werden, die Antike und deren Mythologie als ein Mittel für Kritik am nationalsozialistischen Regime zu benutzen. Hauptmann drückte mit seinen Randnotizen also seine Verblüffung darüber aus, daß die Isaurier - nach seiner Ansicht - nicht nur offenbar das Schicksal der Deutschen vorgelebt, sondern obendrein eine Stadt entsprechenden Namens bewohnt hatten. ${ }^{39}$

35 Clive Foss, s.v. 'Isaura', 'Isaurians' in: $O D B 2$ (1991) 1014.

36 Robert W. Edwards, s.v. 'Isauria', Late Antiquity (1999) $515 f$.

37 Jacob BurckHardT, Die Zeit Constantins des Großen (1853/1880), ND München 1982, 85-88. Hauptmann lag die von Ernst HOHE herausgegebene Erstauflage von 1853 vor.

38 Text + Kritik 142 (1999): Gerhart Hauptmann, 3 ff.

39 Vgl. Sprengel (1999) 7. 